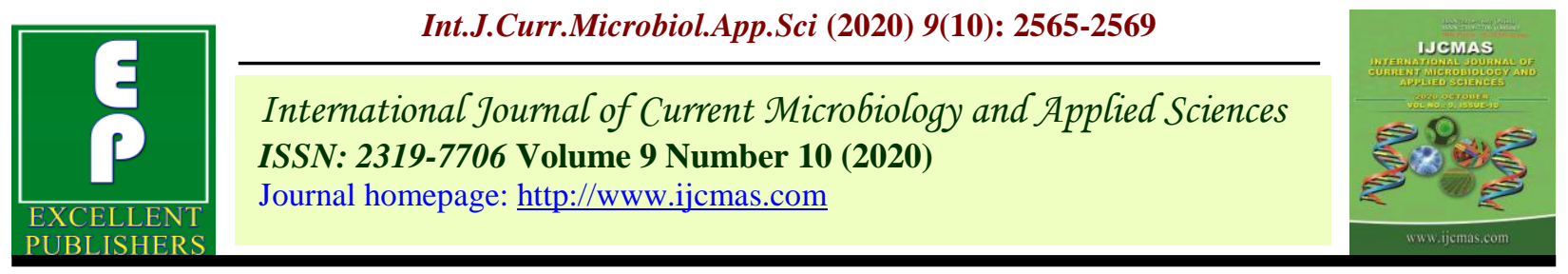

\title{
Effect of Integrated Nutrient Management on Quality and Economics of Beet Root (Beta vulgaris L.) Cv. Crimson Globe under Alkaline Conditions
}

\author{
V. Mounika ${ }^{1 *}$, D. Lakshminarayana ${ }^{2}$, J. Srinivas $^{3}$, G. Sathish ${ }^{4}$ and P. Gouthami ${ }^{5}$
}

${ }^{1}$ Department of Vegetable Science, ${ }^{2}$ Department of Floriculture and Landscaping, College of Horticulture, Rajendranagar, Hyderabad - 500032, ${ }^{3}$ Department of Vegetable Science, College of Horticulture, Mojerla, Wanaparthy -509382, ${ }^{4}$ Department of Agricultural Statistics, College of Horticulture, Mojerla, Wanaparthy-509382, ${ }^{5}$ Department of Crop Physiology, College of Horticulture, Mojerla, Wanaparthy-509382, India

*Corresponding author

\section{A B S T R A C T}

\section{Keywords}

Beet root, Growth parameters and yield parameters

\section{Article Info}

\section{Accepted:}

20 September 2020

Available Online:

10 October 2020
A field experiment was carried out at Vegetable research block, College of HorticultureMojerla, SKLTS Horticultural University, Mulugu, Siddipet, Telangana during rabi season of 2019-20 to study the effect of integrated nutrient management on quality and economics of beetroot (Beta vulgaris 1.) Cv Crimson Globe. The experiment was conducted with three sources of nutrients viz. organic, inorganic and biofertilizers combinations in randomized block design with eight treatments and three replications. Among the treatments, T3 treatment $75 \%$ RDNPK + FYM $\left(6 \mathrm{t} \mathrm{ha}^{-1}\right)+\mathrm{VC}\left(1.5 \mathrm{t} \mathrm{ha}^{-1}\right)+$ Azatobactor $\left(10 \mathrm{~kg} \mathrm{ha}^{-1}\right)+$ PSB $\left(10 \mathrm{~kg} \mathrm{ha}^{-1}\right)$ recorded the highest ascorbic acid content $\left(3.4 \mathrm{mg} 100 \mathrm{~g}^{-1}\right)$, betanine content $\left(1.92 \mathrm{mg} 100 \mathrm{~g}^{-1}\right)$, total soluble solids $\left(9.17^{0}\right.$ brix), highest gross returns (Rs..2,29,920), net returns (Rs..1,83835) and best benefit cost ratio (3.9:1).

\section{Introduction}

Beetroot (Beta vulgaris L.) is one of the important root vegetable crops belongs to the family Chenopodiaceae along with Spinach, Palak, Swiss Chard, Parsley and Celery. It has a diploid chromosome number of $(2 n=18)$ and is native to Western Europe. It produces green tops and a swollen root used both as vegetable and salad, and is grown for food uses (pickles, salad, and juice) rather than for sugar production. The intense red colour of beetroot derives from high concentration of betalains.
Betalains are used in food industry as natural colorants, antioxidant and anti-inflammatory activities are found in betalains so there is an increase attention towards the health benefits of humans (Georgiev et al., 2010 and Zielinska et al., 2009). Betacyanins and betaxanthins are betalains mainly found in beetroot (Gandia et al., 2010). Now a day, beetroot is grown in worldwide and is regularly consumed as a part of the normal diet. It is commonly used in manufacturing as a food colouring agent known as E162 (Clifford et al., 2015). Beetroot juice on 
inflammation is strongly involved in the development and progression of several clinical conditions including coronary heart disease and cancer (Jurgen et al., 2015). Integrated Nutrient Management is an alternative for sustainable crop production rather than use of inorganic fertilizers only. Integrated Nutrient Management (INM) is an approach of supplying nutrition to the crop by including organic and inorganic sources of nutrients. The combined use of organic manures, biofertilizers with a reduced dose of chemical fertilizers, helps to reduce pollution problems, increase the yield and quality of the product and also maintain soil health. Keeping these points in view the present work has been proposed to study the effect of integrated nutrient management on quality and economics of beetroot (Beta vulgaris L.) $\mathrm{Cv}$. Crimson Globe under alkaline conditions.

\section{Material and methods}

The present investigation was conducted at Vegetable Research Block College of Horticulture-Mojerla, SKLTS Horticultural University, Mulugu. The soil was sandy loam texture. The initial soil sample of experimental site had $\mathrm{pH} 7.52$, organic carbon $0.35 \%$ and available $\mathrm{N}, \mathrm{P}$ and $\mathrm{K}$ content of 242.11, 28.2 and $242.3 \mathrm{~kg} \mathrm{ha}^{-1}$ respectively.

The experiment was laid out in a randomized block design with eight treatments having three replications. The total eight treatments consist of i.e. T1-75 \% RDNPK + FYM (12t $\left.\mathrm{ha}^{-1}\right)$ Azatobactor $\left(10 \mathrm{~kg} \mathrm{ha}^{-1}\right)+$ PSB $(10 \mathrm{~kg}$ $\left.\mathrm{ha}^{-1}\right)$, T2-75 \% RDNPK + VC $\left(3 \mathrm{t} \mathrm{ha}^{-1}\right)+$ Azatobactor $\left(10 \mathrm{~kg} \mathrm{ha}^{-1}\right)+$ PSB $\left(10 \mathrm{~kg} \mathrm{ha}^{-1}\right)$, T3-75 \% RDNPK + FYM $\left(6 \mathrm{t} \mathrm{ha}^{-1}\right)+\mathrm{VC}(1.5$ $\left.\mathrm{t} \mathrm{ha}^{-1}\right)+$ Azatobactor $\left(10 \mathrm{~kg} \mathrm{ha}^{-1}\right)+$ PSB (10 $\left.\mathrm{kg} \mathrm{ha}^{-1}\right)$, T4-50 \% RDNPK + FYM (12 $\left.\mathrm{t} \mathrm{ha}^{-1}\right)$ + Azotobactor (10 kg ha $\left.\mathrm{kg}^{-1}\right)+$ PSB (10 $\left.\mathrm{kg} \mathrm{ha}^{-1}\right), \mathrm{T} 5-50 \%$ RDNPK + VC $\left(3 \mathrm{t} \mathrm{ha}^{-1}\right)+$ Azatobactor $\left(10 \mathrm{~kg} \mathrm{ha}^{-1}\right)+$ PSB $\left(10 \mathrm{~kg} \mathrm{ha}^{-1}\right)$, T6-50\% RDNPK+ FYM $\left(6 \mathrm{t} \mathrm{ha}^{-1}\right)+\mathrm{VC}(1.5$ $\left.\mathrm{t} \mathrm{ha}^{-1}\right)+$ Azatobactor $\left(10 \mathrm{~kg} \mathrm{ha}^{-1}\right)+$ PSB (10 $\left.\mathrm{kg} \mathrm{ha}^{-1}\right)$, T7-75 \% RDNPK + FYM $\left(12 \mathrm{t} \mathrm{ha}^{-1}\right)$ and T8-50 \% RDNPK + FYM (12 t ha $\left.{ }^{-1}\right)$. The variety under study was Crinson Globe. Seeds were sown at the spacing of $45 \times 15 \mathrm{~cm}$ and thinning was done 10 days after sowing to maintain spacing. Nitrogen, Phosphorus and potassium were given through Urea, SSP and MOP respectively. Full dose of $\mathrm{P}$ and $\mathrm{K}$ and half dose of $\mathrm{N}$ were applied as basal dose, as per treatment before sowing and remaining half dose of $\mathrm{N}$ was given 30 DAS. Manures viz., farmyard manure and vermicompost were incorporated as per treatment to respective plots prior to sowing. Prior to sowing of seeds, seeds are treated with biofertilizers (Azotobacter and PSB). The data were recorded on five plants per treatment per plot in each replication on growth and yield parameters. Observations were recorded on minimum number of days required for 80 per cent germination of seedlings, plant height, number of leaves per plant, leaf area, chlorophyll content index, root length, root diameter, root yield per plant, root yield per plot, root yield per hectare, root to shoot ratio and harvest index. The data were statistically analysed using analysis of variance (ANOVA) for RBD following the standard procedure as suggested by Panse and Sukhatme (1985).

\section{Results and Discussion}

\section{Quality parameters}

Integrated nutrient management had significant effect on quality parameters and eonomics of beetroot $\mathrm{Cv}$ Crimson Glonbe presented in table 1 and 2 .

\section{Ascorbic acid content (mg $\left.100 \mathrm{~g}^{-1}\right)$}

Among the treatments, $\mathrm{T}_{3}$ treatment $(75 \%$ RDNPK +FYM $\left(6 \mathrm{t} \mathrm{ha}^{-1}\right)+\mathrm{VC}\left(1.5 \mathrm{tha}^{-1}\right)+$ Azatobactor $\left(10 \mathrm{~kg} \mathrm{ha}^{-1}\right)+\operatorname{PSB}\left(10 \mathrm{~kg} \mathrm{ha}^{-1}\right)$ 
recorded significantly maximum ascorbic acid content value (3.4 mg $\left.100 \mathrm{~g} \mathrm{~g}^{-1}\right)$. Similar results were also reported by Meena et al., (2014) in tomato who reported that application of biofertilizers along with vermicompost enhanced the atmospheric nitrogen fixation, resulted in more carbohydrates production led to higher ascorbic acid content. The present investigation was inconsistent with other reports of Kushwah et al., (2019) in radish

\section{Betanine content (mg $100 \mathrm{~g}-1)$}

The data enunciated on betanine content revealed that, $\mathrm{T}_{3}$ treatment $(75 \%$ RDNPK + FYM $\left(6 \mathrm{tha}^{-1}\right)+$ Vermicompost $\left(1.5 \mathrm{t} \mathrm{ha}^{-1}\right)$ + Azatobactor $\left(10 \mathrm{~kg} \mathrm{ha}^{-1}\right)+$ PSB $\left(10 \mathrm{~kg} \mathrm{ha}^{-1}\right)$ recorded the highest value which was due to accumulation of more reserve substances in root portion. The present investigations were inconsistant with other reports of Felczynski and Elkner (2008) in red beet.

\section{Total soluble solids (0 Brix)}

Among the treatments, T3 treatment $(75 \%$ RDNPK +FYM $\left(6 \mathrm{tha}^{-1}\right)+\mathrm{VC}\left(1.5 \mathrm{tha}^{-1}\right)+$ Azatobactor $\left(10 \mathrm{~kg} \mathrm{ha}^{-1}\right)+$ PSB $\left(10 \mathrm{~kg} \mathrm{ha}^{-1}\right)$, recorded the maximum TSS value $\left(9.17^{\circ}\right.$ Brix) which might be due to the same reason studied for the betanine content parameter. The present findings are comparable with that of Sunandarani and Mallareddy (2007) in carrot.

Table.1 Effect of integrated nutrient management on ascorbic acid content ( $\left.\mathrm{mg} 100 \mathrm{~g}^{-1}\right)$ betanine content (mg $\left.100 \mathrm{~g}^{-1}\right)$ and total soluble solids $\left({ }^{0}\right.$ brix) of beet root $\mathrm{Cv}$. Crimson Globe

\begin{tabular}{|c|c|c|c|}
\hline Treatments & $\begin{array}{l}\text { Ascorbic } \\
\text { acid } \\
\text { content (mg } \\
\left.100 \mathrm{~g}^{-1}\right)\end{array}$ & $\begin{array}{c}\text { Betanine } \\
\text { content } \\
\left(\mathrm{mg} 100 \mathrm{~g}^{-1}\right)\end{array}$ & $\begin{array}{c}\text { Total } \\
\text { soluble } \\
\text { solids } \\
\text { ( }{ }^{0} \text { brix) }\end{array}$ \\
\hline $\begin{array}{l}\text { T1-75 \% RDNPK + FYM }\left(12 \mathrm{tha}^{-1}\right)+\text { Azatobactor }\left(10 \mathrm{~kg} \mathrm{ha}^{-1}\right)+\text { PSB } \\
\left(10 \mathrm{~kg} \mathrm{ha}^{-1}\right)\end{array}$ & $2.4^{\mathrm{c}}$ & $1.72^{\mathrm{bc}}$ & $7.40^{c}$ \\
\hline $\begin{array}{l}\mathrm{T}_{2}-75 \% \text { RDNPK + VC }\left(3 \mathrm{t} \mathrm{ha}^{-1}\right)+\text { Azatobactor }\left(10 \mathrm{~kg} \mathrm{ha}^{-1}\right)+\mathrm{PSB}(10 \mathrm{~kg} \\
\left.\mathrm{ha}^{-1}\right)\end{array}$ & $3.2^{\mathrm{ab}}$ & $1.83^{\mathrm{ab}}$ & $8.47^{\mathrm{ab}}$ \\
\hline $\begin{array}{l}\mathrm{T}_{3}-75 \% \text { RDNPK }+ \text { FYM }\left(6 \mathrm{tha}^{-1}\right)+\mathrm{VC}\left(1.5 \mathrm{t} \mathrm{ha}^{-1}\right)+\text { Azatobactor }(10 \mathrm{~kg} \\
\left.\mathrm{ha}^{-1}\right)+ \\
\text { PSB }(10 \mathrm{~kg} \mathrm{ha}-1)\end{array}$ & $3.4^{\mathrm{a}}$ & $1.92^{\mathrm{a}}$ & $9.17^{\mathrm{a}}$ \\
\hline $\begin{array}{l}\text { T4-50\% RDNPK + FYM }\left(12 \mathrm{t} \mathrm{ha}^{-1}\right)+\text { Azotobactor }\left(10 \mathrm{~kg} \mathrm{ha}^{-1}\right)+\text { PSB } \\
\left(10 \mathrm{~kg} \mathrm{ha}^{-1}\right)\end{array}$ & $2.2^{\mathrm{c}}$ & $1.68^{\mathrm{bc}}$ & $7.11^{\mathrm{c}}$ \\
\hline $\begin{array}{l}\text { Ts-50\% RDNPK + VC }\left(3 \mathrm{tha}^{-1}\right)+\text { Azatobactor }\left(10 \mathrm{~kg} \mathrm{ha}^{-1}\right)+\mathrm{PSB}(10 \\
\left.\mathrm{kg} \mathrm{ha}^{-1}\right)\end{array}$ & $2.2 \mathrm{c}$ & $1.77_{\mathrm{ab}}$ & $8.13 b$ \\
\hline $\begin{array}{l}\text { T6-50 \% RDNPK+ FYM }\left(6 \mathrm{t} \mathrm{ha}^{-1}\right)+\mathrm{VC}\left(1.5 \mathrm{t} \mathrm{ha}^{-1}\right)+\text { Azatobactor }(10 \mathrm{~kg} \\
\left.\mathrm{ha}^{-1}\right)+ \\
\text { PSB }(10 \mathrm{~kg} \mathrm{ha}-1)\end{array}$ & $3.1^{b}$ & $1.84^{\mathrm{ab}}$ & $8.70^{\mathrm{ab}}$ \\
\hline T7-75 \% RDNPK + FYM $\left(12 \mathrm{t} \mathrm{ha}^{-1}\right)$ & $2.4 \mathrm{c}$ & $1.68 \mathrm{bc}$ & $6.74_{\mathrm{cd}}$ \\
\hline $\mathrm{T}_{8}-50 \%$ RDNPK + FYM $\left(12 \mathrm{t} \mathrm{ha}^{-1}\right)$ & $2.1^{\mathrm{c}}$ & $1.58^{\mathrm{c}}$ & $6.31^{d}$ \\
\hline SEm \pm & 0.11 & 0.24 & 0.24 \\
\hline CD at $5 \%$ & 0.32 & 0.71 & 0.71 \\
\hline
\end{tabular}


Table.2 Effect of integrated nutrient management on economics of beet root Cv. Crimson Globe

\begin{tabular}{|c|c|c|c|c|}
\hline Treatments $(\mathrm{T})$ & $\begin{array}{l}\text { Total cost } \\
\quad \text { of } \\
\text { cultivation } \\
\left(\mathrm{Rs} . \mathrm{ha}^{-1}\right)\end{array}$ & $\begin{array}{l}\text { Gross } \\
\text { returns( } \\
\left.\text { ₹ } \text { ha }^{-1}\right)\end{array}$ & $\begin{array}{l}\text { Net } \\
\text { Returns } \\
\left(₹ \text { ha }^{-1}\right)\end{array}$ & $\begin{array}{l}\mathrm{B}: \mathrm{C} \\
\text { ratio }\end{array}$ \\
\hline $\begin{array}{l}\text { T1-75 \% RDNPK + FYM }\left(12 \mathrm{tha}^{-1}\right)+\text { Azatobactor }\left(10 \mathrm{~kg} \mathrm{ha}^{-1}\right)+ \\
\text { PSB }\end{array}$ & 44,585 & $1,89,000$ & $1,44,415$ & 3.23 \\
\hline \multicolumn{5}{|l|}{$\left(10 \mathrm{~kg} \mathrm{ha}^{-1}\right)$} \\
\hline $\begin{array}{l}\text { T2-75\% RDNPK + VC }\left(3 \mathrm{t} \mathrm{ha}^{-1}\right)+\text { Azatobactor }\left(10 \mathrm{~kg} \mathrm{ha}^{-1}\right)+ \\
\text { PSB (10kg }\end{array}$ & 48,585 & $2,10,480$ & $1,61,895$ & 3.33 \\
\hline \multicolumn{5}{|l|}{$\left.\mathrm{ha}^{-1}\right)$} \\
\hline $\begin{array}{l}\text { T3-75 \% RDNPK + FYM }\left(6 \mathrm{tha}^{-1}\right)+\mathrm{VC}\left(1.5 \mathrm{t} \mathrm{ha}^{-1}\right)+\text { Azatobactor } \\
(10 \mathrm{~kg}\end{array}$ & 46,085 & $2,29,920$ & 1,83835 & 3.9 \\
\hline \multicolumn{5}{|l|}{ ha-1) + PSB (10 kg ha-1) } \\
\hline $\begin{array}{l}\text { T4-50 \% RDNPK + FYM }\left(12 \mathrm{tha}^{-1}\right)+\text { Azotobactor }\left(10 \mathrm{~kg} \mathrm{ha}^{-1}\right)+ \\
\text { PSB }\end{array}$ & 42,251 & $17,19,60$ & $1,29,709$ & 3.06 \\
\hline \multicolumn{5}{|l|}{$\left(10 \mathrm{~kg} \mathrm{ha}^{-1}\right)$} \\
\hline T5-50 \% RDNPK + VC $\left(3 \mathrm{t} \mathrm{ha}^{-1}\right)+$ Azatobactor $\left(10 \mathrm{~kg} \mathrm{ha}^{-1}\right)+$ & 45,851 & $1,90,800$ & $1,44,949$ & 3.16 \\
\hline $\begin{array}{l}\text { PSB }(10 \\
\left.\mathrm{kg} \mathrm{ha}^{-1}\right)\end{array}$ & & & & \\
\hline $\begin{array}{l}\text { T6-50\% RDNPK+ FYM }\left(6 \mathrm{tha}^{-1}\right)+\mathrm{VC}\left(1.5 \mathrm{tha}^{-1}\right)+\text { Azatobactor } \\
(10 \mathrm{~kg}\end{array}$ & 45,751 & $2,20,920$ & $1,75,169$ & 3.82 \\
\hline \multicolumn{5}{|l|}{$\left.\mathrm{ha}^{-1}\right)+\operatorname{PSB}\left(10 \mathrm{~kg} \mathrm{ha}^{-1}\right)$} \\
\hline T7-75 \% RDNPK + FYM $\left(12 \mathrm{t} \mathrm{ha}^{-1}\right)$ & 40,585 & $1,51,200$ & $1,10,615$ & 2.72 \\
\hline T8-50 \% RDNPK + FYM $\left(12 \mathrm{t} \mathrm{ha}^{-1}\right)$ & 34,117 & $1,23,720$ & 89,603 & 2.62 \\
\hline
\end{tabular}

\section{Economics}

From the table 2 T3 treatment (75\% RDNPK + FYM $\left(6 \mathrm{t} \mathrm{ha}^{-1}\right)+$ Vermicompost $(1.5$ ha-1) + Azatobactor (10 kg ha-1) + PSB (10 kg ha-1) recorded the highest gross returns (₹ $2,29,920)$, net returns (₹ $1,83,835)$ and benefit cost ratio (3.9:1), which was due to the same treatment recorded higher root yield per hectare over other treatments. These findings are in line with the reports of Shanu et al., (2019) in carrot.

\section{References}

Clifford, T., Stevenson, E. J., Howatson, G. and West, D. J. 2015. The potential benefits of red beetroot supplementation in health and disease. Journal of Nutrients. 7: 2801- 2822.

Felczynski K., Elkner K. 2008. Effect of longterm organic and mineral fertilization on yield and quality of red beet (Beta vulgaris L.). Veget. Crops Res. Bull. 68: 111-125.

Gandia, F., Escribano, J and Garcia, F. 2010. Structural implications on color, fluorescence, and antiradical activity in betalains. An International Journal of Plant Biology. 232(2): 449-460.

Georgiev, V. G., Weber, J., Kneschke, E. M., Denev, P. N., Bley, T and Pavlov, A. I. 2010. Antioxidant activity and phenolic content of betalain extracts from intact 
plants and hairy root cultures of the red beetroot (Beta vulgaris). Journal of Plant Foods for Human Nutrition. 65(2): 105-111.

Jurgen, W., Gundula, W., Stefan, H., Pinar, U., Peter, $\mathrm{L}$ and Ulrike, M. 2015.Compositional characteristics of commercial beetroot products and beetroot juice prepared from seven beetroot varieties grown in Upper Austria. Journal of Food Composition and Analysis. 42: 46-55.

Kushwah, G.; Sharma, R. K.; Kushwah, S.S. and Mishra, S. N. (2019).Effect of organic manures, inorganic fertilizers and varieties on growth, yield and quality of tropical carrot. Indian Journal of Horticulture 76(3): 451-456.

Meena, R.K., Kumar, S., Maji, S., Kumar, D and Kumar, M. 2014. Effect of organic manures and biofertilizers on growth, flowering, yield and quality of tomato cv. Pusa Sheetal. International Journal of Agricultural Sciences 10(1):329-332.

Panse, V. G. and Sukhatme, P. V. 1957. Publication and information division,
Statistical Indian methods for agricultural workers. Council of Agricultural Research.New Delhi. pp. 87-89.

Shanu, V., D. Lakshminarayana, P. Prasanth and Saidaiah, D. 2019. Studies on the Effect of integrated nutrient management (INM) on growth and yield parameters of carrot (Daucas carota L.) cv. Kuroda Improved under Southern Telangana Conditions. Int.J.Curr.Microbiol.App.Sci. $\quad$ 8(04): 2786-2791.

SunandaRani, N and Mallareddy, K. 2007. Effect of different organic manures and inorganic fertilizers on growth, yield and quality of carrot (Daucus carota L.). Karnataka Journal of Agricultural Sciences. 20 (3): 686-688.

Zielinska, P. M., Olejnik, A., Dobrowolska, Z and Grajek, W. 2009. In vitro effects of beetroot juice and chips on oxidative metabolism and apoptosis in neutrophils from obese individuals. Phytotherapy Research. 23(1):49-55

\section{How to cite this article:}

Mounika, V., D. Lakshminarayana, J. Srinivas, G. Sathish and Gouthami, P. 2020. Effect of Integrated Nutrient Management on Quality and Economics of Beet Root (Beta vulgaris L.) Cv. Crimson Globe under Alkaline Conditions. Int.J.Curr.Microbiol.App.Sci. 9(10): 25652569. doi: https://doi.org/10.20546/ijcmas.2020.910.308 